\title{
Molecular Mechanism of Bone Marrow Mesenchymal Stem Cell Derived Exosomes on Vascular Repair in Rats with Brain Injury
}

JIEHUA MA*, MIN CAO ${ }^{1}$, L. SUN, Y. ZHAO, YANLI HUO AND H. ZHANG

Basic Medical College, Hebei North University, Zhangjiakou 075000, 'Department of Pathology, Beijing Liangxiang Hospital, Beijing 102401, China

Ma et al.: Mechanism of Bone Marrow Mesenchymal Stem Cell Derived Exosomes

To explore the molecular mechanism of exosomes derived from bone marrow mesenchymal stem cells on vascular repair in rats with brain injury. 30 Sprague-Dawley rats were used in this study. Bone marrow was extracted from the posterior iliac crest of the rat under general anesthesia and bone marrow mesenchymal stem cells were isolated and cultured. Some rats were treated with middle cerebral artery occlusion surgery to induce acute brain injury models. The protein expressions of vascular endothelial growth factor $A$ and vascular endothelial growth factor receptor 2 were analyzed by western blot. Polymerase chain reaction was used to analyze the messenger RNA expressions of endothelin-1, vascular cell adhesion molecule 1 and endothelial nitric oxide synthase related to endothelial cell damage. The protein expressions of vascular endothelial growth factor $A$ and vascular endothelial growth factor receptor 2 in the model group was lower than that in the control group $(\mathbf{p}<0.05)$ and the protein expressions in the treatment group was higher than that in the model group $(\mathbf{p}<\mathbf{0 . 0 5})$. The messenger RNA expressions of endothelin-1 and vascular cell adhesion molecule 1 in the model group increased while the expression of endothelial nitric oxide synthase messenger RNA decreased $(p<0.05)$. The messenger RNA expressions of endothelin-1 and vascular cell adhesion molecule 1 in the treatment group decreased and the messenger RNA expression of endothelial nitric oxide synthase increased $(p<0.05)$. The apoptosis level of endothelial cells in the model group increased and in the treatment group was reduced. Exosomes derived from bone marrow mesenchymal stem cells could effectively regulate cell survival, promote angiogenesis, protect nerve cells and improve the prognosis of stroke in rats by up regulating the expression of $\mathrm{C}$-X-C chemokine receptor type 4 in bone marrow mesenchymal stem cells.

Key words: Bone marrow mesenchymal stem cells, exosomes, brain injury, vascular repair

Stroke is an acute cerebrovascular disease caused by local cerebral blood circulation disorder, which is the second leading cause of human death in the world $^{[1]}$. Brain micro vascular endothelium is a thin layer of connecting and anchoring dependent cells, which constitutes the interface between blood and solid vessel wall ${ }^{[2]}$. This vascular endothelium is a highly active metabolic system, which synthesizes various vascular regulatory factors to regulate the microcirculation of brain tissue ${ }^{[3]}$. Brain micro vascular endothelial cells are now considered as a highly active metabolic system, which synthesizes various substances to nourish nerves and regulate vascular motor function. As we all know, effective collateral circulation can protect brain tissue after cerebral ischemia by increasing blood perfusion in ischemic penumbra ${ }^{[4]}$. It has been proved that micro vascular integrity and cerebrovascular regeneration can stabilize brain perfusion, thus promoting neuron survival, brain plasticity and nerve recovery ${ }^{[5]}$. Therefore, enhancing angiogenesis in ischemic brain tissue is considered as a new opportunity for potential therapeutic strategies. Bone marrow mesenchymal stem cells (BMSCs) can secrete a variety of nutritional factors and promote cell repair and growth. Many studies have shown that stem cells can inhibit inflammation in ischemic areas and

*Address for correspondence

E-mail: majiehua1981@163.com 
promote angiogenesis ${ }^{[6]}$. Therefore, stem cell therapy is considered as one of the most promising treatments for ischemic stroke. C-X-C chemokine receptor type 4 (CXCR4) belongs to $\mathrm{G}$ protein-coupled receptor super family. Vascular germination requires migration and polarization of endothelial cells in response to the fact that the exosomes of cytokines are small cell membrane vesicles with a diameter of 30-100 $\mathrm{nm}^{[7]}$. Extracellular body is the carrier of intercellular signal transmission and plays an important role in intercellular communication. More and more evidences show that mesenchymal stem cells can promote vascular remodeling in ischemic area after cerebral ischemia. However, it is not clear whether the absent exosomes in CXCR4 over expressed BMSCs play a role in angiogenesis. In this study, we investigated the role of exosomes of BMSCs over expressed in CXCR4 in restoring vascular function and nerve repair after ischemic cerebral infarction and clarified the mechanism of exosomes promoting angiogenesis and protecting endothelial cells, thus providing new ideas for the treatment of stroke and further mechanism research.

\section{MATERIALS AND METHODS}

\section{Experimental materials:}

Experimental animals: 30 Sprague-Dawley (SD) rats (female, weighed $120 \pm 20 \mathrm{~g}$, aged $6 \mathrm{w}$ ) were purchased from Beijing Charles River Experimental Animal Technology Co., Ltd. Rats were kept in a clean room with controllable temperature and light, with light/dark time of $12 \mathrm{~h}$, temperature of $19-23^{\circ}$ and humidity of 40-60\%. The diet and drinking water of the rats were not restricted. A total of 5 rats were raised in each cage.

Isolation and culture of BMSCs: Bone marrow (BM) was extracted from bilateral posterior iliac crest of rats under general anesthesia. $30 \mathrm{ml} \mathrm{BM}$ was collected with $10 \mathrm{ml}$ syringe and heparin sodium was used as anticoagulant. Enriched monocytes were separated on Ficoll Paque Premium (Pennsylvania, USA) by density gradient centrifugation. After Ficoll was isolated, mononuclear cells were inoculated into T75 cell culture bottle, coated with CTSTM CELLstartTM matrix solution (diluted in phosphate buffered saline (PBS) at a ratio of 1:300), with a density of $22.5 \times 10^{6}$ cells/ bottle. Cells were expanded in MesenCultTM ACF Plus medium (Vancouver, Canada) at $5 \% \mathrm{CO}_{2}$ and $37^{\circ}$. After $48 \mathrm{~h}$, a new medium was used instead of the medium containing non adherent cells for cell growth. When the fusion rate of cells reached $80 \%$, cells were divided into the next generation with $0.05 \%$ trypsin.
Middle cerebral artery occlusion in rats: Rats were anesthetized by intraperitoneal injection of 1 $\%$ pentobarbital sodium. 6-0 coated silicone nylon monofilament suture (Beijing Zhongneng Technology Co., Ltd., China) was introduced into the external carotid artery cavity and was gently pushed into the internal carotid artery to block the origin of the middle cerebral artery. For rats in sham operation group, suture was inserted into the lumen of external carotid artery but was not pushed into the internal carotid artery. After 90 min of occlusion, the suture was withdrawn to allow reperfusion. The neurological deficit score was evaluated by 5 scale and the rats with scores between 1 and 3 were considered as middle cerebral artery occlusion (MCAO) success.

Lentiviral transfection: The backbone (P-glycoprotein (Pgp)) of lentiviral vector was purchased from Shanghai Novo Biotechnology Co., Ltd. At first, Pgp-Sir and Pgp-null were transiently transfected into 293T cells by pPACKH1-human immunodeficinecy virus (HIV) lentivirus packaging kit (American system Bioscience Company) to produce a large number of viruses. Secondly, the virus supernatant was isolated and purified and added to the $3^{\text {rd }}$ to $6^{\text {th }}$ generation of BMSCs for culture for $72 \mathrm{~h}$. Lentivirus was transfected at different multiplicity of infection (MOI) values $(10,30$, 60,80 and 100) and the most suitable MOI value was determined by immunofluorescence. Finally, lentivirus genome was integrated into BMSCs genome by virus transfection with $\mathrm{MOI}=80$. Transfection efficiency was detected by immunofluorescence. BMSCs were divided into two groups: BMSCs transfected with blank lentiviral vector (BMSCCon) and BMSCs transfected with CXCR4 lentiviral vector (BMSCCXR4).

Experimental grouping: The rats were divided into the following groups: control group (rats without arterial occlusion receiving sham operation, $n=10$ ), model group (established acute middle cerebral artery occlusion model, $n=10$ ) and treatment group (rats in model group were injected with exosomes derived from BMSCCXCR4, $\mathrm{n}=10$ ). Cultured cells were divided into bEnd.3 group (separately cultured bEnd.3 was used as blank control), bEnd.3+exosome group (co-incubation with BMSCCXCR4 exosome), bEnd.3+exosome+inhibitor group (inhibition of BMSCCXCR4 after addition of AMD3100).

Medical ethics issues: All procedures involving animals are carried out in accordance with the regulations of the agency's Animal Care and Use Committee. 


\section{Experimental methods:}

Western blot: Aortic tissues of all rats were extracted with radioimmunoprecipitation assay buffer (RIPA) lysis buffer. Protein concentration assay was carried out using bicinchoninic acid (BCA) protein analysis kit and $35 \mu \mathrm{g}$ protein was analyzed using $10 \%$ sodium dodecyl sulfate polyacrylamide gel electrophoresis (SDSPAGE). The protein was transferred to polyvinylidene fluoride (PVDF) membrane. The membrane was treated in blocking solution (5\% skim milk) for $2 \mathrm{~h}$ at room temperature and incubated with anti-vascular endothelial growth factor A (VEGFA) (1:1000; cat. No.: A0686; ABclonal Biotechnology Co., Ltd.), antipoly (ADP-ribose) polymerase (anti-PARP) (1:1000; cat. ABclonal Biotechnology Co., Ltd.), anti-vascular endothelial growth factor receptor 2 (anti-VEGFR2) (1: 1000; cat. ABclonal Biotechnology Co., Ltd.), antiB-cell lymphoma 2 (anti-Bcl-2) (1:1000; cat. ABclonal Biotechnology Co., Ltd.), anti-Bcl-2-associated X protein (anti-BAX) label (1:1000; cat. no.50430-2AP; ProteinTech Group, Inc.) or anti- $\beta$-actin antibody (1: 5000; cat. No.20536-1-AP; ProteinTech Group, Inc.). The membrane was kept overnight at $4^{\circ}$. After that, it was incubated with horseradish peroxidase (HRP) goat anti-rabbit immunoglobulin G (IgG) $(\mathrm{H}+\mathrm{L})$ secondary antibody (1:5000; cat. No.: AS014; ABclonal Biotechnology Co., Ltd.) and let stand at room temperature for $1 \mathrm{~h}$. Then, it was detected with enhanced chemiluminescence (ECL) detection reagent (equilibrium and equilibrium molecular dynamics (EMD) micropore). Optical density of the obtained band was measured using ImageJ $2 \times$ software (Rawak software, Inc.) and the density measurement value was normalized to $\beta$-actin.

Quantitative reverse transcription polymerase chain reaction (qRT-PCR): Total ribonucleic acid (RNA) was extracted from aortic tissue by TRIzol ${ }^{\circledR}$. According to the manufacturer's instructions (15 min at $37^{\circ}$ and $5 \mathrm{~s}$ at $85^{\circ}$ ), complementary deoxyribonucleic acid (cDNA) was synthesized from RNA using cDNA first strand synthesis kit (Takara Bio, Inc.). The qRTPCR was performed using SYBR Green-PCR master mixture (Takara-Bio, Inc.). The qRT-PCR reaction amplification system is: initial denaturation at $95^{\circ}$ for $10 \mathrm{~min}$, denaturation at $95^{\circ}$ for 40 times for $15 \mathrm{~s}$, annealing at $60^{\circ}$ for $15 \mathrm{~s}$ and extension at $60^{\circ}$ for 60 s. The reaction was carried out in polymerase chain reaction (PCR) amplification system (ABI 7300). The expression data of related genes were analyzed by $2^{-\Delta \Delta \mathrm{Cq}}$ method.
Triphenyltetrazolium chloride (TTC) staining method: Rats were anesthetized by intraperitoneal injection of $2 \%$ pentobarbital sodium $3 \mathrm{~d}$ after ischemia/ reperfusion (I/R) and their brains were taken after intragastric administration (NS). Coronal slicing of brain was carried out with a brain slicer at a distance of $2 \mathrm{~mm}$ from the frontal pole. The slices were incubated with $1 \%$ TTC solution at $37^{\circ}$ for $15 \mathrm{~min}$ in the dark and soaked in $4 \%$ paraformaldehyde.

Modified nerve severity score: On the $3^{\text {rd }}$ and $14^{\text {th }} \mathrm{d}$ after $\mathrm{I} / \mathrm{R}$, the rats were examined with modified nerve severity scores (mNSS). The evaluation was conducted by blind method. mNSS includes exercise (muscle state, abnormal movement), sensation (vision, touch and proprioception), reflex and balance tests. Scores range from 0 to a maximum of 14. Evaluation of nerve function defect degree: 1-5 points, mild defect; 6-9 points, moderate defect; 10-14 points, severe defect.

Flow cytometry: BMSCs were washed twice with PBS and $1 \sim 5 \times 10^{5}$ cells were collected. $1 \times$ binding buffer was added to resuspend the cells and the cell concentration was adjusted to $10^{6} / \mathrm{ml}$ cells. After mixing well, the mixture reacted in the dark for $15 \mathrm{~min}$ at room temperature. CELLQuest software was used to analyze various data of flow cytometer to adjust forward scattered light (FSC), side scattered light (SSC) and voltage; fluorescence compensation was adjusted according to standard fluorescein; percentage of positive cells was detected.

Detection of cell proliferation by cell counting kit-8 (CCK-8): Three types of cells were inoculated in a 96well plate at a density of $1 \times 10^{4}$ cells/well and 5 wells were set as a group. In the experiment of promoting the proliferation of bEnd. 3 cells by corpuscles in vitro, bEnd. 3 cells were added with fresh medium containing $10 \%$ CCK 8 solution according to the concentration of corpuscles and cultured in an incubator with $5 \% \mathrm{CO}_{2}$ saturation humidity at $37^{\circ}$ for 0.5 to $4 \mathrm{~h}$. The optical density (OD) value of bEnd.3 cells at $450 \mathrm{~nm}$ was detected by a microplate reader.

Pipe formation test: Cells were cultured in medium with or without exosomes for $24 \mathrm{~h}$. Cells in each group were inoculated into 48 -well plates $\left(5 \times 10^{3}\right.$ cells per well), coated with matrix glue, with 3 holes in each group. After $6 \mathrm{~h}$, the morphogenesis of vascular tube was observed with inverted optical microscope under 100 times magnifying glass. ImageJ software was used to record the number of vascular lumen structures in five random fields. 
Terminal deoxynucleotidyl transferase dUTP nick end labeling (TUNEL) analysis: TUNEL assay kit was used as described above (cat. No.: 11684817910; Roche Diagnostics). Tissue samples were fixed in $4 \%$ formaldehyde at room temperature for $24 \mathrm{~h}$ and embedded in paraffin. Next, a paraffin section with a thickness of $4 \mu \mathrm{m}$ was adhered to the glass slide. Slices were dewaxed by heating the slides at $60^{\circ}$ for $30 \mathrm{~min}$. After that, they were washed twice with PBS. Samples were added to $50 \mu 1$ TUNEL analytical solution and incubated at $37^{\circ}$ for $60 \mathrm{~min}$ in the dark and were then washed twice with PBS. Slices were counterstained with hematoxylin at room temperature for 5-10 min to observe the nuclei. The membrane was sealed by antifluorescence quenching method and the slices were observed under a $400 \times$ confocal microscope.

\section{Statistical analysis:}

Statistical package for the social sciences (SPSS) 20.0 software (IBM Company, New York, USA) and GraphPad Prism 7.0 (GraphPad Software Company, California, USA) were used for data analysis; all data were expressed as mean \pm standard deviation (SD). $\mathrm{p}<0.05$ indicated statistically significant difference.

\section{RESULTS AND DISCUSSION}

Analysis of expression of angiogenic protein is carried out. The expressions of VEGFA and VEGFR2 in the model group were lower than that in the control group $(p<0.05)$, while those in the treatment group were higher than those in the model group $(p<0.05)$. Exocrine therapy promoted angiogenesis (fig. 1 and Table 1).

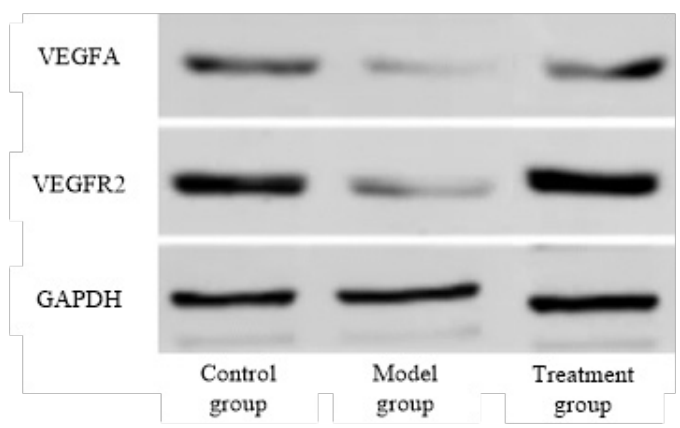

Fig. 1: Western blot analysis of the expressions of angiogenic factors

TABLE 1: WESTERN BLOT ANALYSIS OF THE

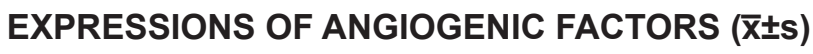

\begin{tabular}{lcc}
\hline Group & VEGFA & VEGFR2 \\
\hline Control group & $2.14 \pm 0.25$ & $1.95 \pm 0.23$ \\
Model group & $1.16 \pm 0.13$ & $1.07 \pm 0.08$ \\
Treatment group & $1.92 \pm 0.22$ & $1.84 \pm 0.20$ \\
F value & 11.402 & 13.115 \\
p value & 0.011 & 0.005 \\
\hline
\end{tabular}

RT-PCR analysis of messengerRNA (mRNA) expressions of endothelial cell injury-related factors was found. The mRNA expressions of endothelin-1 (ET-1), vascular cell adhesion molecule 1 (VCAM1) and endothelial nitric oxide synthase (eNOS) in endothelial cells were analyzed by PCR. Compared with the control group, the mRNA expressions of ET-1 and VCAM-1 in the model group were higher and the mRNA expression of eNOS was lower $(p<0.05)$ (Table 2).

TABLE 2: PCR ANALYSIS OF EXPRESSIONS OF ET-1, VCAM-1 AND ENOS ( $\bar{x} \pm s)$

\begin{tabular}{lccc}
\hline Group & ET-1 & VCAM-1 & eNOS \\
\hline Control group & $1.08 \pm 0.09$ & $1.15 \pm 0.11$ & $2.24 \pm 0.23$ \\
Model group & $2.16 \pm 0.23$ & $2.05 \pm 0.20$ & $1.15 \pm 0.9$ \\
Treatment & $1.35 \pm 0.14$ & $1.26 \pm 0.13$ & $1.93 \pm 0.18$ \\
group & 13.936 & 11.625 & 10.384 \\
F value & 0.011 & 0.003 & 0.027 \\
p value &
\end{tabular}

Exosomes reduced the area of cerebral infarction in rats. TTC staining was used to calculate and compare the infarct volume of rats. On the $3^{\text {rd }}$ and $14^{\text {th }} \mathrm{d}$, the infarct volume of the model group was larger than that of the control group $(\mathrm{p}<0.05)$, while that of the treatment group was smaller than that of the model group $(\mathrm{p}<0.05)$ (Table 3$)$.

TABLE 3: MEASUREMENT OF CEREBRAL INFARCTION VOLUME IN RATS ( $\overline{\mathrm{X}} \pm \mathrm{s})$

\begin{tabular}{lcc}
\hline Group & $3 \mathrm{~d}$ & $14 \mathrm{~d}$ \\
\hline Control group & $3.15 \pm 0.15$ & $3.37 \pm 0.20$ \\
Model group & $35.46 \pm 4.38$ & $27.82 \pm 4.64$ \\
Treatment group & $17.75 \pm 1.66$ & $14.02 \pm 2.18$ \\
F value & 11.402 & 13.115 \\
$\mathrm{p}$ value & 0.032 & 0.015 \\
\hline
\end{tabular}

The nerve injury of rats was compared by improved mNSS score. It was found that the score of the model group was higher than that of the control group $(\mathrm{p}<0.05)$, while the score of the treatment group was lower than that of the model group $(\mathrm{p}<0.05)$. Exocrine treatment reduced the nerve damage caused by brain injury (Table 4).

TABLE 4: MNSS SCORE OF RATS ( $\overline{\mathrm{x}} \pm \mathrm{s})$

\begin{tabular}{lcc}
\hline Group & $3 \mathrm{~d}$ & $14 \mathrm{~d}$ \\
\hline Control group & $1.36 \pm 0.05$ & $1.24 \pm 0.03$ \\
Model group & $6.58 \pm 0.46$ & $4.73 \pm 0.32$ \\
Treatment group & $3.17 \pm 0.25$ & $2.64 \pm 0.28$ \\
F value & 11.402 & 13.115 \\
p value & 0.020 & 0.005 \\
\hline
\end{tabular}


Exosomes inhibited apoptosis. The expressions of apoptosis-related factors Bcl-2, Bax and PARP were analyzed by western blot. Compared with the control group, the protein expressions of Bcl-2 and PARP in the model group decreased, while the protein expressions of Bcl-2 and PARP in the treatment group increased and the Bax expression decreased. Apoptosis of endothelial cells was detected by TUNEL method. The apoptotic cells showed green fluorescence under fluorescence microscope. Compared with the control group, the apoptosis level of endothelial cells in the model group increased. Compared with the model group, the apoptosis of endothelial cells in the treatment group decreased, indicating that BMSCs could repair arterial injury by inhibiting the apoptosis of endothelial cells (fig. 2 and Table 5).

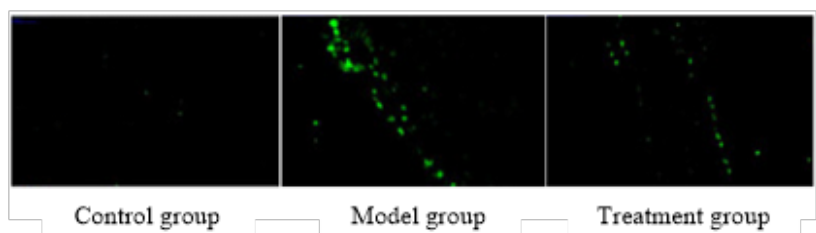

Fig. 2: TUNEL detection of apoptosis

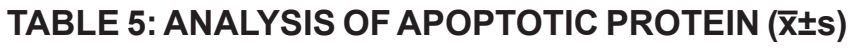

\begin{tabular}{lccc}
\hline Group & Bcl-2 & Bax & PARP \\
\hline Control group & $2.15 \pm 0.24$ & $1.18 \pm 0.11$ & $1.93 \pm 0.20$ \\
Model group & $1.18 \pm 0.09$ & $2.23 \pm 0.26$ & $1.05 \pm 0.07$ \\
Treatment & $1.86 \pm 0.21$ & $1.34 \pm 0.13$ & $1.83 \pm 0.16$ \\
group & 9.825 & 11.237 & 10.463 \\
F value & 0.014 & 0.022 & 0.003 \\
p value &
\end{tabular}

Exosomes promoted the proliferation of cerebrovascular endothelial cells. Cell proliferation and apoptosis were analyzed by CCK-8 and 5-ethynyl-2'-deoxyuridine (EdU) fluorescence staining. Compared with bEnd.3 group, bEnd.3+exosome group had higher cell proliferation and lower cell apoptosis rate $(\mathrm{p}<0.05)$, while bEnd.3+exosome+inhibitor group had lower cell proliferation and higher cell apoptosis rate $(\mathrm{p}<0.05)$ (Table 6).

TABLE 6: ANALYSIS OF CELL PROLIFERATION

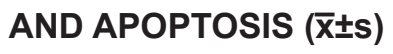

\begin{tabular}{lcc}
\hline Group & $\begin{array}{c}\text { Cell } \\
\text { proliferation }\end{array}$ & $\begin{array}{c}\text { Cell apoptosis } \\
\text { (\%) }\end{array}$ \\
\hline bEnd.3 group & $1.14 \pm 0.05$ & $21.15 \pm 3.66$ \\
bEnd.3+exosome group & $1.86 \pm 0.15$ & $11.54 \pm 1.25$ \\
bEnd.3+exosome+inhibitor & $1.25 \pm 0.08$ & $27.62 \pm 4.29$ \\
group & 10.493 & 12.058 \\
F value & 0.025 & 0.011 \\
p value & & \\
\hline
\end{tabular}

Role of exosomes in endothelial cell tube formation is shown here. As shown in fig. 3, compared with the bEnd. 3 group, the bEnd.3+exosomes could promote the angiogenesis of endothelial cells $(\mathrm{p}<0.05)$. The addition of AMD 3100 would seriously affect the formation of tubes (fig. 3).

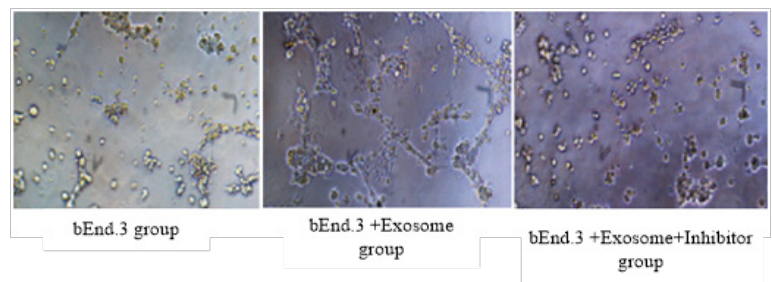

Fig. 3: Exosomes promoted angiogenesis of endothelial cells Hypoxic-ischemic brain injury is a complex process, including early and late biological pathways. Among the involved pathways, angiogenesis and neurogenesis are the main targets for treating stroke at present ${ }^{[8]}$. According to reports, mesenchymal stem cells (MSCs) transplantation is a candidate target for the treatment of neonatal hypoxic-ischemic brain damage, which attracts more and more attention from stroke researchers. Exosomes derived from MSCs mediate the therapeutic effects of MSCs. Exosomes are vesicles packaged by cell membranes and used for information transmission ${ }^{[9]}$. Therefore, it can be speculated that by increasing CXCR4 on cell membrane, the number of CXCR4 on exosomes can be increased. In this study, BMSCs were genetically modified by lentiviral transfection to overexpress CXCR4 and exosomes secreted by BMSCs were isolated.

In vivo experiment, TTC staining was used to measure the volume of cerebral infarction in rats to determine the successful conduction of MCAO model. MNSS score and debonding test were used to evaluate the recovery of MCAO rats. The experimental results showed that injection of exogenous CXCR4 could accelerate the recovery of MCAO rats and the exogenous CXCR4 group recovered fastest. It is suggested that CXCR4 may promote the active role of exosomes in ischemiareperfusion injury. In vitro experiments, exocrine CXCR4 could promote the formation of brain microvascular endothelial cells. These effects were all offset by CXCR4 inhibitor AMD3100. This evidence not only proves that BMSC exosomes can promote angiogenesis after ischemia, but also shows that CXCR4 can improve this effect.

BMSCs can obviously inhibit inflammation. In previous studies, BMSCs have been injected into animals with central nervous system damage and it is found that BMSCs can survive and migrate into damaged tissues 
and finally improve the neural function ${ }^{[10]}$. After ischemic stroke forms blood clots, endothelial cells die due to lack of nutrition, which destroys blood-brain barrier, causes edema of brain tissue and aggravates disease progress ${ }^{[11]}$. Promoting the formation of new blood vessels in ischemic area is of great significance to improve the prognosis of cerebral infarction. Some data show that $21 \mathrm{~d}$ after ischemia, new blood vessels are formed at the edge of the ischemic area and gradually extend to the ischemic center through budding ${ }^{[12]}$. Studies have shown that CXCR4 can promote the stability of endothelial cells and improve the prognosis of atherosclerotic diseases through anti-apoptotic proteins in vivo ${ }^{[13]}$. Cobalt chloride $\left(\mathrm{CoCl}_{2}\right)$ can cause hypoxia, leading to autophagy and apoptosis. In this study, model group rats increased the expression of pro-apoptotic protein Bax and decreased the expression of anti-apoptotic protein Bcl-2 and PARP, while treatment group could reverse the effect of MCAO, which indicated that exosome CXCR4 could reduce apoptosis. Eccrine cells derived from BMSCs are helpful to repair vascular degeneration caused by abdominal aortic aneurysm. Nitric oxide (NO) and ET-1 are antagonistic vasoactive substances synthesized and secreted by vascular endothelial cells. ET-1 negatively regulates the release of NO in endothelial cells and the increase of NO concentration inhibits the formation of ET-1. The dynamic balance between them maintains the normal vascular endothelial function and the increase of ET-1 and the decrease of NO are the signs of vascular endothelial injury ${ }^{[14]}$. ENOS can synthesize NO. In previous studies, there were many factors in vivo and in vitro accelerating or delaying the process of reendothelialization by up-regulating or down-regulating the expression of eNOS. PCR analysis showed that the mRNA expressions of ET-1 and VCAM-1 in the model group were higher than those in the control group, while the expression of eNOS mRNA was lower. In the treatment group, the expression trend in model group was reversed.

The new data support the beneficial effects of angiogenesis and neurovascular repair and the close interaction between angiogenesis and neurogenesis. Ischemic stroke is characterized by ischemic core, with rapid neuronal death and a vulnerable infarct surrounding area, while the angiogenic blood vessels near the infarct act as the scaffold of nerve progenitor cells and enable neurons to migrate to the ischemic surrounding area ${ }^{[15]}$. Therefore, neurogenesis and angiogenesis can be regarded as complex dynamic coupling between neurons and endothelial cells through VEGF, neurotrophins and their homologous receptors on neurons and endothelial cells. According to recent reviews, cell-based and cell-free therapies (exosomes, extracellular vesicles, microRNAs) have been successfully applied to angiogenesis-mediated tissue regeneration with great potential in the treatment of ischemic heart disease, stroke and bone defect ${ }^{[16]}$. In addition, exosomatic derived communication between endothelial cells and cardiomyocytes shows the potential to induce local neovascularization during heart injury ${ }^{[17,18]}$. In this study, we found that the protein expressions of angiogenic factors VEGFA and VEGFR2 decreased in MCAO rats, which was related to the decrease of angiogenesis and the regulation of VEGFA and VEGFR2 was released. In addition, injection of BMSCs-derived exosomes containing CXCR4 mimetics could improve the inhibition of angiogenesis in MCAO rats, which further supported exosomes as potential factors for treating hypoxic-ischemic brain damage.

To sum up, our research showed that exosomes derived from BMSCs could effectively regulate cell survival, promote angiogenesis, protect nerve cells and improve the prognosis of stroke in rats by up-regulating the expression of CXCR4 in BMSCs.

\section{Acknowledgements:}

The research is supported by Youth Fund Project of Education Department of Hebei province (No. QN2019080)

\section{Conflicts of interest:}

The authors declared no conflict of interest.

\section{REFERENCES}

1. Chang CL, Chen HH, Chen KH, Chiang JY, Li YC, Lin HS, et al. Adipose-derived mesenchymal stem cell-derived exosomes markedly protected the brain against sepsis syndrome induced injury in rat. Am J Transl Res 2019;11(7):3955-71.

2. Li C, Fei K, Tian F, Gao C, Song Y. Adipose-derived mesenchymal stem cells attenuate ischemic brain injuries in rats by modulating miR-21-3p/MAT2B signaling transduction. Croat Med J 2019;60(5):439-48.

3. Chang Q, Hao Y, Wang Y, Zhou Y, Zhuo H, Zhao G. Bone marrow mesenchymal stem cell-derived exosomal microRNA125a promotes M2 macrophage polarization in spinal cord injury by downregulating IRF5. Brain Res Bull 2021;170:199210.

4. Ghosh S, Garg S, Ghosh S. Cell-derived exosome therapy: a novel approach to treat post-traumatic brain injury mediated neural injury. ACS Chem Neurosci 2020;11(14):2045-7. 
5. Zhang Y, Wang WT, Gong CR, Li C, Shi M. Combination of olfactory ensheathing cells and human umbilical cord mesenchymal stem cell-derived exosomes promotes sciatic nerve regeneration. Neural Regen Res 2020;15(10):1903-11.

6. Williams AM, Wu Z, Bhatti UF, Biesterveld BE, Kemp MT, Wakam GK, et al. Early single-dose exosome treatment improves neurologic outcomes in a 7-day swine model of traumatic brain injury and hemorrhagic shock. J Trauma Acute Care Surg 2020;89(2):388-96.

7. Williams AM, Bhatti UF, Brown JF, Biesterveld BE, Kathawate RG, Graham NJ, et al. Early single-dose treatment with exosomes provides neuroprotection and improves bloodbrain barrier integrity in swine model of traumatic brain injury and hemorrhagic shock. J Trauma Acute Care Surg 2020;88(2):207-18.

8. Xiong L, Sun L, Zhang Y, Peng J, Yan J, Liu X. Exosomes from bone marrow mesenchymal stem cells can alleviate early brain injury after subarachnoid hemorrhage through miRNA129-5pHMGB1 pathway. Stem Cells Dev 2020;29(4):212-21.

9. Nalamolu KR, Venkatesh I, Mohandass A, Klopfenstein JD, Pinson DM, Wang DZ, et al. Exosomes secreted by the cocultures of normal and oxygen-glucose-deprived stem cells improve post-stroke outcome. Neuromolecular Med 2019;21(4):529-39.

10. Zhao Y, Gan Y, Xu G, Yin G, Liu D. MSCs-derived exosomes attenuate acute brain injury and inhibit microglial inflammation by reversing CysLT2R-ERK1/2 mediated microglia M1 polarization. Neurochem Res 2020;45(5):1180-90.

11. Medalla M, Chang W, Calderazzo SM, Go V, Tsolias A, Goodliffe JW, et al. Treatment with mesenchymal-derived extracellular vesicles reduces injury-related pathology in pyramidal neurons of monkey perilesional ventral premotor cortex. J Neurosci 2020 ;40(17):3385-407.

12. Tsintou M, Dalamagkas K, Moore TL, Rathi Y, Kubicki M, Rosene DL, et al. The use of hydrogel-delivered extracellular vesicles in recovery of motor function in stroke: a testable experimental hypothesis for clinical translation including behavioral and neuroimaging assessment approaches. Neural Regen Res 2021;16(4):605-13.
13. Zhao H, Li Y, Chen L, Shen C, Xiao Z, Xu R, et al. HucMSCsderived miR-206-knockdown exosomes contribute to neuroprotection in subarachnoid hemorrhage induced early brain injury by targeting BDNF. Neuroscience 2019;417:1123.

14. Guo S, Perets N, Betzer O, Ben-Shaul S, Sheinin A, Michaelevski I, et al. Intranasal delivery of mesenchymal stem cell derived exosomes loaded with phosphatase and tensin homolog siRNA repairs complete spinal cord injury. ACS Nano 2019;13(9):10015-28.

15. Xian P, Hei Y, Wang R, Wang T, Yang J, Li J, et al. Mesenchymal stem cell-derived exosomes as a nanotherapeutic agent for amelioration of inflammation-induced astrocyte alterations in mice. Theranostics 2019;9(20):5956-75.

16. Williams AM, Dennahy IS, Bhatti UF, Halaweish I, Xiong Y, Chang P, et al. Mesenchymal stem cell-derived exosomes provide neuroprotection and improve long-term neurologic outcomes in a swine model of traumatic brain injury and hemorrhagic shock. J Neurotrauma 2019;36(1):54-60.

17. Kong LY, Liang MY, Liu JP, Lai P, Ye JS, Zhang ZX, et al. Mesenchymal stem cell-derived exosomes rescue oxygenglucose deprivation-induced injury in endothelial cells. Curr Neurovasc Res 2020;17(2):155-63.

18. Kazemi E, Zargooshi J, Kaboudi M, Heidari P, Kahrizi D, Mahaki B, et al. A genome-wide association study to identify candidate genes for erectile dysfunction. Brief Bioinform 2020 .

This is an open access article distributed under the terms of the Creative Commons Attribution-NonCommercial-ShareAlike 3.0 License, which allows others to remix, tweak, and build upon the work non-commercially, as long as the author is credited and the new creations are licensed under the identical terms

This article was originally published in a special issue,
"Therapeutic Perspectives in Biomedical Research and Pharma-
ceutical Sciences and their Nursing Methods"
Indian J Pharm Sci 2021:83(4)Spl issue "214-220"

